\title{
Crystalline Polymer Ultrathin Films from Mesoscopic Precursors**
}

\author{
Qiong Tong, Marina Krumova, and Stefan Mecking*
}

Thin and ultrathin crystalline polymer films are of funda mental scientific as well as practical interest concerning their structures and properties. ${ }^{[16]}$ For the preparation of ultrathin $(\leq 0.1 \mu \mathrm{m})$ films the polymer is applied to the substrate in a non ordered state, usually as a dilute solution in an organic solvent. To overcome the intra and intermolecular interac tions responsible for crystalline order in the solid polymer, and render it soluble, high temperatures are often required. Crystalline order only forms on the substrate during solvent removal or cooling. As a different approach to ultrathin films of crystalline polymers we report on their preparation at room temperature from aqueous dispersions of prefabricated polymer nanocrystals functioning as building blocks (Scheme 1). The very small particle size, in combination with the basic phenomenon that the amorphous regions are located at the surface exclusively in polymer single crystals, results in an efficient interaction between particles in films.

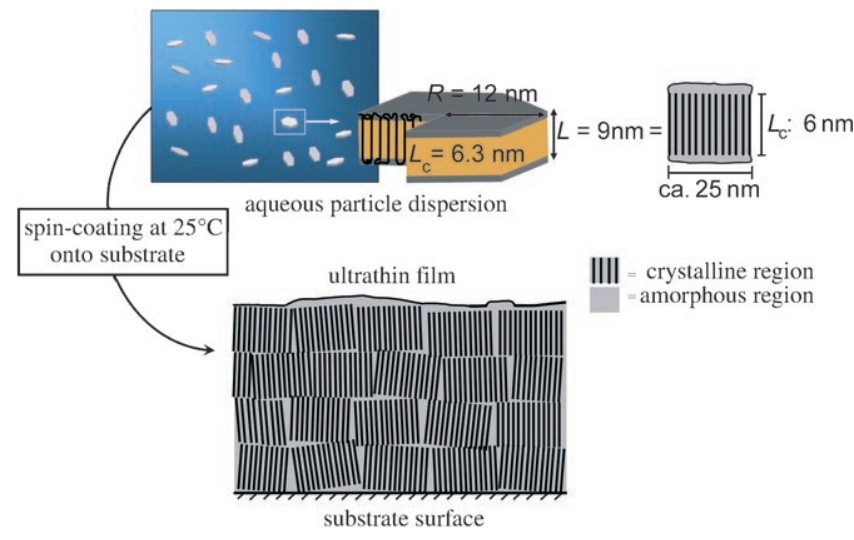

Scheme 1. Film preparation and film structure (surfactant molecules adsorbed to the surface of dispersed particles not shown for the sake of clarity).

The construction of materials from individual nanoscale crystalline entities as building blocks has been studied intensely for inorganic materials. Here, this method has been employed as a route to mesoscopic materials, that is,

[*] Q. Tong, Dr. M. Krumova, Prof. Dr. S. Mecking

University of Konstanz

Chair of Chemical Materials Science

Dept. of Chemistry

Universitätsstrasse 10, 78467 Konstanz (Germany)

Fax: $(+49) 7531885152$

E mail: stefan.mecking@uni konstanz.de

Homepage: http://www.chemie.uni konstanz.de/agmeck/

[**] Financial support by the DFG (international research training group "soft condensed matter") and by the BMBF (project 03X5505) is gratefully acknowledged. S.M. is indebted to the Fonds der Chemischen Industrie and to the Hermann Schnell Foundation. materials intermediate in order between glasses or liquids and bulk inorganic crystals, but with a more complex, often hierarchical structure than the latter. ${ }^{[7]}$ Also, the occurrence of such entities as intermediates is discussed to be a general principle of inorganic crystal formation. ${ }^{[8]}$ In our case, the nanoscale building blocks consist of an organic polymer, and their order results from the principles of polymer crystalliza tion, which includes the presence of amorphous phases as a general feature of a crystal.

Polyethylene is the simplest organic polymer in terms of molecular structure, and it is of vast practical importance. Its crystallization and structure in the bulk have been inves tigated intensely. ${ }^{[9]}$ Also, studies of single crystals of polyeth ylene have been ground breaking for the understanding of polymer crystallization. ${ }^{[10,11]}$ These studies typically employed isolated crystals with several micrometers lateral extension prepared by crystallization from supersaturated solutions in organic solvents. ${ }^{[12]}$ Ultrathin polyethylene films have been prepared previously by evaporating solutions in hydrocarbon solvents at temperatures of 100 to $180^{\circ} \mathrm{C}^{\left[\begin{array}{ll}13 & 15\end{array}\right]}$

Aqueous dispersions of nanoscale polyethylene crystals can be obtained by catalytic polymerization of ethylene with water soluble nickel(II) complexes. ${ }^{[16]}$ These dispersions are essentially separated, nonaggregated single crystals (lateral size, that is, pseudodiameter ca. $25 \mathrm{~nm}$ ) consisting of a single crystalline lamella (thickness $6 \mathrm{~nm}$ ) covered by thin amor phous layers (thickness ca. $1 \mathrm{~nm}$ ). ${ }^{[17]}$

For the present study, polyethylene particles were employed as $2 \mathrm{wt} \%$ aqueous dispersions containing just enough surfactant (sodium dodecyl sulfate, SDS) to colloi dally stabilize the particles. A surface tension of $65 \mathrm{mN} \mathrm{m}^{-1}$ demonstrates that virtually all surfactant is adsorbed on the particles. The polyethylenes studied were highly linear (less than 5 branches per 1000 carbon atoms ${ }^{[18]}$ ) and had number average molecular weights $M_{\mathrm{n}}$ of approximately $2 \times$ $10^{5} \mathrm{~g} \mathrm{~mol}^{-1}$ and molecular weight distributions $M_{\mathrm{w}} / M_{\mathrm{n}}$ of around 2 . Their melting points $T_{\mathrm{m}}$ in the dispersed particles ${ }^{[19]}$ are around $127^{\circ} \mathrm{C}\left(T_{\mathrm{m}}=132^{\circ} \mathrm{C}\right.$ for isolated bulk polymer $)$. Dropwise application of the aqueous nanoparticle dispersions to a glass slide and subsequent drying at room temperature affords transparent films, which appear homogeneous by optical microscopy (glass was chosen as a substrate because of its planarity and optical transparency). These films are about $1 \mu \mathrm{m}$ thick. To obtain ultrathin films, spincoating was employed. Coverage of the substrate without holes or defects is evident from AFM (Figure 1). The surface roughness amounts to approximately $5 \mathrm{~nm}$ as concluded from line scans over several micrometers. A uniform thickness of the films is also confirmed by ellipsometry. Film thicknesses are typically around $50 \mathrm{~nm}$, as found independently by ellipsometry, AFM of purposefully introduced scratches in the film, and energy loss in TEM (see below). 

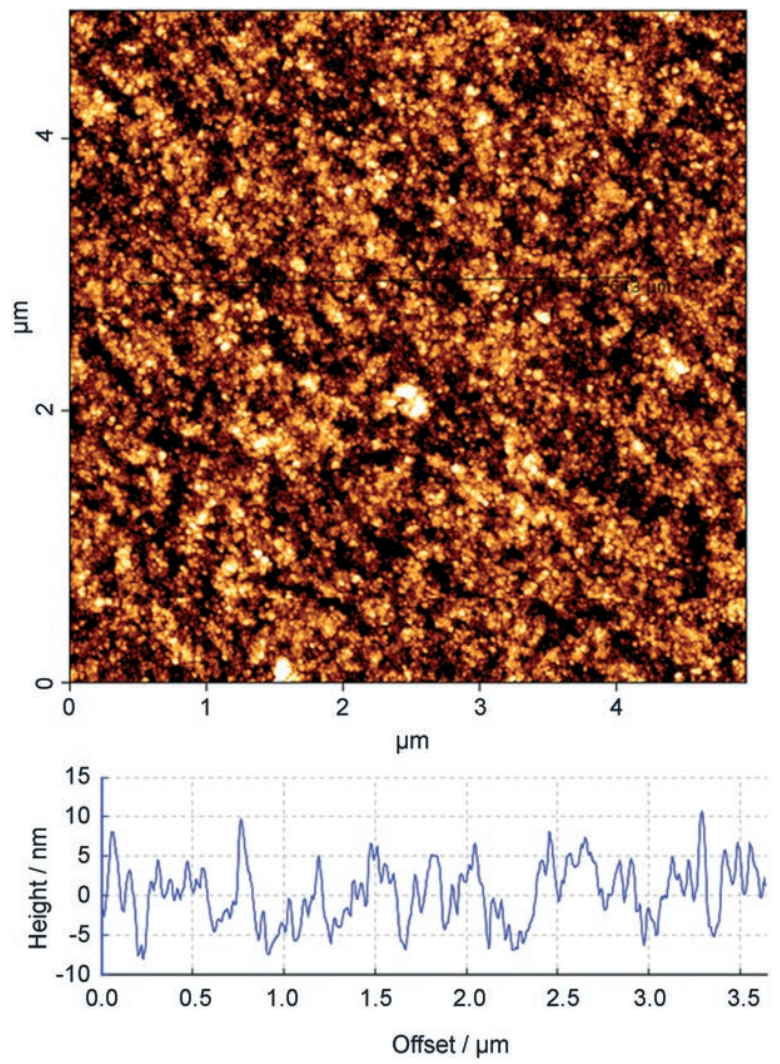

Figure 1. AFM image of ultrathin film prepared from crystalline nano particles.

For TEM studies, the films were floated off the glass slide onto water. Free standing films were obtained (Figure 2). Analysis of the films by electron energy loss spectroscopy (EELS) and studies of water contact angles give no indication of the presence of surfactant, which is removed during the floating off procedure (see the Experimental Section). TEM shows an overall homogeneous structure of the films and variations of the electron density on a local scale (Figure 2, left). In accordance with the absence of larger crystalline structures, the films appear dark when viewed with cross polarizers in an optical microscope. For comparison, after annealing of films above the melting point and subsequent cooling to room temperature, open spherulitic structures with

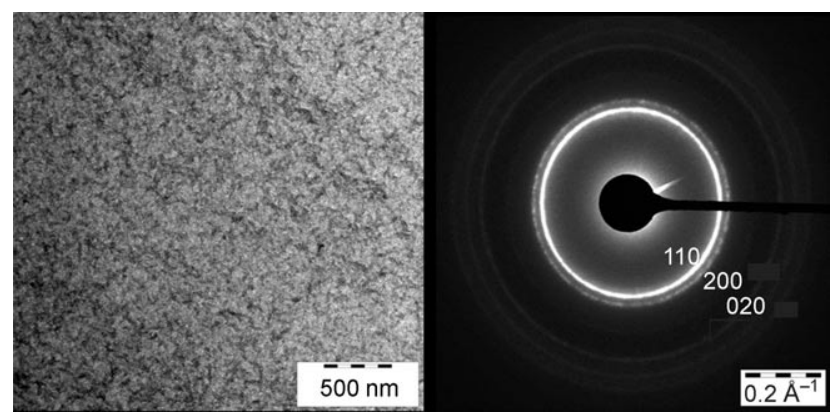

Figure 2. TEM image of a free standing film (left), and electron diffraction pattern with the electron beam perpendicular to the film surface (right). sizes of several micrometers are found by AFM and optical microscopy as expected. ${ }^{[13,14]}$

Electron diffraction on the nascent spin coated films demonstrates the presence of crystalline phases, which consist of the most common orthorhombic polyethylene lattice unit. With the incident beam direction perpendicular to the film surface, sharp $(h k 0)$ diffraction rings are observed (Figure 2, right), which indicates that the crystals are oriented prefer entially with their lamella $c$ axis perpendicular to the sub strate surface (Scheme 1). ${ }^{[20,21]}$ The differences in electron density on a local scale observed for films (Figure 2, left) can be attributed to some degree of disorientation of the platelets in the films.

In summary, construction from individual crystalline mesoscale entities is an attractive method for the generation of crystalline polymer ultrathin films. The combination of a very small crystal size with the general structure of polymer single crystals results in efficient interaction through the amorphous parts despite their overall minor volume share. This situation has been demonstrated for linear polyethylene, a high volume commodity plastic that is also physiologically inert and environmentally benign, but difficult to process to thin and ultrathin films to date. It is an inherent feature of the reported approach that it is carried out at ambient temper atures and does not involve hazardous and toxic organic solvents, which can also be beneficial for sensitive substrates. The "nascent" film structures, and the interaction of the primary particle building blocks at elevated temperatures, but below their melting point may also be of interest with respect to current dispute over the role of mesophases in polymer crystallization. $\left.^{[22} 25\right]$

\section{Experimental Section}

Dispersion synthesis: Aqueous polymer nanoparticle dispersions ${ }^{[17]}$ were prepared according to reference [16]. In brief, an aqueous surfactant containing solution of the water soluble catalyst precursor $\left[\left\{\kappa^{2} N, O 6 \mathrm{C}(\mathrm{H}) \mathrm{N}\left(2,6\left\{3,5\left(\mathrm{~F}_{3} \mathrm{C}\right)_{2} \mathrm{C}_{6} \mathrm{H}_{3}\right\}_{2} \mathrm{C}_{6} \mathrm{H}_{3}\right) 2,4\right.\right.$

$\left.\left.\mathrm{I}_{2} \mathrm{C}_{6} \mathrm{H}_{2} \mathrm{O}\right\} \mathrm{NiMe}(\mathrm{L})\right]$ ( $\mathrm{L}$ di or trisulfonated triphenylphosphine) was exposed to ethylene at $40 \mathrm{~atm}$ and $15^{\circ} \mathrm{C}$ for $30 \mathrm{~min}$. The degree of branching (from high temperature ${ }^{13} \mathrm{C}$ NMR spectroscopy), molec ular weight (from high temperature size exclusion chromatography vs. linear polyethylene standards), and bulk thermal properties (from DSC, $10 \mathrm{~K} \mathrm{~min}^{-1}$ ) were determined on bulk polymer, obtained by precipitating an aliquot of dispersion with excess methanol and drying in vacuo.

Preparation of films: Polished glass slides were cleaned by immersing in a 7:3 mixture of $96 \% \mathrm{H}_{2} \mathrm{SO}_{4}$ and $30 \% \mathrm{H}_{2} \mathrm{O}_{2}$ at $80^{\circ} \mathrm{C}$ for around $30 \mathrm{~min}$, rinsed thoroughly with distilled water in an ultrasonic bath, and dried in air. Freshly cleaved mica was used without further treatment. Polyethylene dispersions were spin coated on the sub strates. A drop of dispersion was placed on the resting substrate, which was then accelerated at a rate of $300 \mathrm{rpm} \mathrm{s}^{-1}$ to a final speed of $2000 \mathrm{rpm}$. The nascent films are transparent. For TEM, the as prepared film on a glass or mica substrate was inserted into distilled water at an angle of approximately $45^{\circ}$. The floated off film was transferred onto a copper grid and dried at room temperature overnight prior to TEM. In comparison to a reported value of around $90^{\circ}$ for polyethylene,${ }^{[26]}$ the static water contact angle on the surface of nascent films was determined to be approximately $15^{\circ}$ for spin coated films (and also for thicker films obtained by drying several drops of dispersion on a glass slide and subsequent removal of any 
residual water in vacuo). This difference probably originates from accumulation of the surfactant on the film surface during film formation. ${ }^{[27]}$ However, most of the surfactant can be removed by washing the film surface with water, or floating off from the substrate, respectively, as indicated by an increase of the contact angle to $95^{\circ}$ after washing. No sulfur, indicative of SDS surfactant, is detected by EELS.

Microscopy: The topography of the films was characterized with a JPK NanoWizard atomic force microscope in the intermittent contact mode using a Silicon tip with a force constant of $40 \mathrm{Nm}^{-1}$ and resonant frequency of about $300 \mathrm{kHz}$. Height and phase images were recorded simultaneously. To minimize the deformation of the film surface by the scanning probe, the set point amplitude $A_{s p}$ was chosen about 0.70 .8 of the free oscillating amplitude $A_{0}$. TEM images were obtained on a Zeiss Libra 120 instrument operated at $120 \mathrm{kV}$ on unstained samples.

Received: March 3, 2008

Published online: May 16, 2008

Keywords: mesoscopic materials · polyethylene · polymer single crystals · ultrathin films

[1] J. Sommer, G. Reiter, Adv. Polym. Sci. 2006, 200, 136

[2] G. Krausch, R. Magerle, Adv. Mater. 2002, 14, 15791583.

[3] G. Wegner, Thin Solid Films 1992, 216, 105116.

[4] W. Zhou, X. Weng, S. Jin, S. Rastogi, A. J. Lovinger, B. Lotz, S. Z. D. Cheng, Macromolecules 2003, 36, 94859491.

[5] V. H. Mareau, R. E. Prud'Homme, Soft Materials (Eds.: J. R. Dutcher, A. G. Marangoni), Marcel Dekker, New York, 2005, pp. 3971

[6] G. Reiter, I. Botiz, L. Graveleau, N. Grozev, K. Albrecht, A. Mourran, M. Möller, Lect. Notes Phys. 2007, 714, 179200.

[7] M. Antonietti, G. Ozin, Chem. Eur. J. 2004, 10, 2841.

[8] H. Cölfen, S. Mann, Angew. Chem. 2003, 115, 2452 2468; Angew. Chem. Int. Ed. 2003, 42, 2350 2365. .

[9] G. R. Strobl, The Physics of Polymers, 3rd ed., Springer, Berlin, 2007.
[10] A. Keller, Philos. Mag. 1957, 2, 11711175.

[11] E. W. Fischer, Z. Naturforsch. A 1957, 12, 753754.

[12] Although isolated single crystals were in the focus of these studies, it has been noted that the micrometer sized polymer single crystals can aggregate to form a compact thick film during slow filtering of their suspensions in organic solvents: A. Keller, A. O'Connor, Nature 1957, 180, 12891290.

[13] Z. Bartczak, A. S. Argon, R. E. Cohen, T. Kowalewski, Polymer 1999, 40, 23672380.

[14] O. Mellbring, S. Kihlman Oiseth, A. Krozer, J. Lausmaa, T. Hjertberg, Macromolecules 2001, 34, 74967503.

[15] Y. Wang, S. Ge, M. Rafailovich, J. Sokolov, Y. Zhou, H. Ade, J. Lüning, A. Lustiger, G. Marom, Macromolecules 2004, 37, 3319 3327.

[16] I. Göttker Schnetmann, B. Korthals, S. Mecking, J. Am. Chem. Soc. 2006, 128, 77089.

[17] C. H. M. Weber, A. Chiche, G. Krausch, S. Rosenfeldt, M. Ballauff, L. Harnau, I. Göttker Schnetmann, Q. Tong, S. Meck ing, Nano Lett. 2007, 7, 20242029.

[18] These few branches present are methyl groups, which have a low impact on the crystallinity. ${ }^{[9]}$

[19] Q. Tong, M. Krumova, I. Göttker Schnetmann, S. Mecking, Langmuir 2008, 24, 23412347.

[20] B. K. Vainshtein, Structure Analysis by Electron Diffraction, Pergamon, Oxford, 1964.

[21] Electron diffraction was also performed with the incident beam inclined to the film normal direction by tilting the sample. The sharp (110) and (200) diffraction rings (Figure 2, right) change to continuous arcs, as expected. ${ }^{[20]}$

[22] S. Rastogi, H. Hikosaka, M. Kawabata, A. Keller, Macromole cules 1991, 24, 63846391.

[23] E. B. Sirota, A. B. Herhold, Science 1999, 283, 529532.

[24] G. Strobl, Prog. Polym. Sci. 2006, 31, 398442.

[25] E. B. Sirota, Macromolecules 2007, 40, 10431048.

[26] W. Xu, P. Liu, H. Li, X. Xu, J. Appl. Polym. Sci. 2000, 78, 243 249.

[27] C. L. Zhao, Y. Holl, T. Pith, M. Lambla, Colloid Polym. Sci. 1987, 265,823829 